\title{
Discovery of cell-type specific DNA motif grammar in cis-regulatory elements using random Forest
}

\author{
Xin Wang ${ }^{1,2}$, Peijie Lin ${ }^{1,2}$ and Joshua W. K. Ho ${ }^{1,2^{*}}$ \\ From 16th International Conference on Bioinformatics (InCoB 2017) \\ Shenzhen, China. 20-22 September 2017
}

\begin{abstract}
Background: It has been observed that many transcription factors (TFs) can bind to different genomic loci depending on the cell type in which a TF is expressed in, even though the individual TF usually binds to the same core motif in different cell types. How a TF can bind to the genome in such a highly cell-type specific manner, is a critical research question. One hypothesis is that a TF requires co-binding of different TFs in different cell types. If this is the case, it may be possible to observe different combinations of TF motifs - a motif grammar - located at the TF binding sites in different cell types. In this study, we develop a bioinformatics method to systematically identify DNA motifs in TF binding sites across multiple cell types based on published ChIP-seq data, and address two questions: (1) can we build a machine learning classifier to predict cell-type specificity based on motif combinations alone, and (2) can we extract meaningful cell-type specific motif grammars from this classifier model.

Results: We present a Random Forest (RF) based approach to build a multi-class classifier to predict the cell-type specificity of a TF binding site given its motif content. We applied this RF classifier to two published ChIP-seq datasets of TF (TCF7L2 and MAX) across multiple cell types. Using cross-validation, we show that motif combinations alone are indeed predictive of cell types. Furthermore, we present a rule mining approach to extract the most discriminatory rules in the RF classifier, thus allowing us to discover the underlying cell-type specific motif grammar.
\end{abstract}

Conclusions: Our bioinformatics analysis supports the hypothesis that combinatorial TF motif patterns are cell-type specific.

Keywords: DNA motif, Transcription factor, Random Forest, Cell-type specificity, Cis-regulatory element

\section{Background}

Transcription factors (TFs) are proteins which usually bind to genomic DNA at specific DNA sequences (motifs) [1]. The binding of different TFs to DNA is critical for the regulation of gene expression in almost all important biological processes, including embryogenesis [2-4], cell cycle and development control [5-7], and response to intercellular signals and environment $[8,9]$. We already know different TFs can be added as exogenous reprogramming factors to convert somatic cells to

\footnotetext{
* Correspondence: j.ho@victorchang.edu.au

'Victor Chang Cardiac Research Institute, Darlinghurst, NSW 2010, Australia ${ }^{2}$ St. Vincent's Clinical School, University of New South Wales, Darlinghurst, NSW 2010, Australia
}

other cell types (e.g., fibroblasts to pluripotent cells) [10]. One interesting observation is that many TFs bind to genomic DNA at different loci depending on the cell-type and biological context (such as signalling pathway activation) in which the TF is expressed, even though the TF binds to the same core motif across different cell types and conditions. For example, Frietze et al. found that the same TF (TCF7L2) can bind to different genomic loci across different cell types [11]. One hypothesis is that any particular TF requires the co-binding of different combions of cell-type specific TFs, such as master regulators [12], in different cell-types [13]. If this is the case, it may be possible to observe different combination of motifs $-\mathrm{a}$ 
motif grammar - being present in the binding sites of the same TF across different cell types.

Several studies have attempted to explore this hypothesis using a computational synthetic biology approach $[14,15]$. Furthermore, spatial co-occurrence patterns of specific pairs of motifs have also been systematically investigated computationally [16]. Some other studies have investigated the questions of cell-type specificity using a combination of sequence features, chromatin structure as well as histone modification marks $[17,18]$. These works all lend evidence to the assumption that a DNA motif grammar - a set of rules based on combinations of TF motifs - in cis-regulatory module exists, and is celltype specific.

To further test this motif grammar hypothesis, we wish to explore the use of a machine learning classifier to predict the cell-type specificity of a TF binding site. Several published studies have used a similar machine learning approach to explore cell-type specificity, including a SVM classification model using $k$-mer sequences in TF ChIP-seq peaks, histone modifications and DNase accessibility data as features to train and predict cell-type specific TF binding sites [19]; a computational approach that considers celltype specific histone H3K27ac DNA profiles around transcription start sites with neural network to predict gene expression in mouse embryonic stem cells [20]; and a deep neural network approach to predict the sequence specificities of DNA and RNA-binding proteins [21].

Nonetheless, we wish to explore a specific question that more directly tests the motif grammar hypothesis can we build a multi-class machine learning classifier based on combinations of sequence motifs alone? Furthermore, we note that many machine learning classifiers that have good performance (e.g., SVM, deep neural network) are hard to visualise and interpret. In other words, it is often difficult to extract the rules - the grammar - from these machine learning models. In this work, we propose to address these issues by developing a Random Forest (RF) based multi-class cell-type classifier based on TF motif combinations alone, and use a recently developed rule-mining approach to extract important discriminatory rules from the trained RF classifier.

Random Forest, first published as random decision forests [22], is a machine learning algorithm for classification and regression. A RF consists of a collection of decision trees, where each tree consists of a random subsample of features [23]. Random Forest could measure feature importance by calculating the 'mean decrease accuracy'. Compared to classification based on an individual decision tree, a RF has been shown to be more robust against the overfitting problem [24]. With the ability to perform multi-class classification, and generally having superior performance, RF is widely used in various fields of biological and biomedical research [25-29]. One short-coming of RF is that it is commonly considered to be a 'black box' machine learning method, as it is not easy to extract and visualise the decision rules that lead to a particular prediction. Recently, some methods have been developed to extract interpretable rules from a RF [30], and software tools have been developed to extract, trim and prune importance rules in a RF [31].

In the following study, we use this RF approach to analyse two published ChIP-seq TF datasets (TCF7L2 and MAX) from ENCODE [32]. Our finding reveals that combinatorial DNA motifs can be cell-type specific, and that we can extract biologically meaningful motif grammars from a RF classifier.

\section{Results}

Identification of cell-type specific cis-regulatory elements The TF ChIP-seq datasets we tested by RF were downloaded from the ENCODE project dataset [32] (see Additional file 1). Data for TF proteins TCF7L2 (Transcription factor 7-like 2) and MAX (myc-associated factor $\mathrm{X}$ ) were chosen because they were systematically profiled across a good number of cell-types (6 cell types in TCF7L2 and 5 cell types in MAX). TCF7L2, which at a downstream effector of the Wnt singling pathway, is a TF that affects the transcription of a variety of genes that affect a diverse set of biological functions [33, 34]. It is also linked to human diseases, including type 2 diabetes [35-37] and a variety of cancers [33, 38, 39]. MAX is a TF protein that is able to form homo-dimers or hetero-dimers with other proteins, which include MYC, MXL1 and MAD [40]; these dimers promote cell differentiation and apoptosis [40]. Many reports also showed that MAX is related to the small cell lung cancer (SCLC) [41-43].

For the TCF7L2 dataset, there are six human cell-lines, including colon cancer cells (HCT116), embryonic kidney cells (HEK293), cervical carcinoma cells (HeLa-S3), liver cancer cells (HepG2), mammary gland adenocarcinoma cells (MCF-7) and pancreatic cancer cells (PANC-1). For the MAX dataset, the five human cell-lines are adenocarcinomic alveolar basal epithelial cells (A549), lymphoblastoid cells (GM12878), immortalised myelogenous leukaemia cells (K562), HeLa-S3 and HepG2.

We extracted the 500 strongest unique ChIP-seq peaks by $p$-value from each cell-line in both the TCF7L2 and MAX datasets (see Additional file 2). We have developed a pipeline to extract the DNA sequences at these ChIPseq peaks, and identified known motifs (based on a large motif database from ENCODE [44]) in these sites using a DNA motif annotation pipeline (see Methods). We were able to extract the number of occurrences of each motif in these sites. This becomes the training set for our RF classifier. The predictive ability of our RF using the TCF7L2 and MAX datasets is evaluated using cross- 
validation. Furthermore, the final trained RF classifier is mined to extract meaningful rules. Figure 1 illustrates the workflow of our DNA motif annotation and RF analysis pipeline.

\section{Combinations of motifs are predictive of cell-types}

We applied 10 times 10-fold Cross-Validation (CV) to evaluate the ability of RF to classify TF sites from different cell-types based on motif occurrence patterns. In order to determine the optimal size of binding site in the two datasets, we first investigate the effect of varying the size of the binding site from $+/-5 \mathrm{bp}$ around the peak centre to $+/-300 \mathrm{bp}$ around the peak centre. We found that the best prediction accuracy can be achieved when the TF binding site is $\sim 240$ bp in length (120 bp up and downstream from the peak centre; see Additional file 3). Therefore,we use $240 \mathrm{bp}$ around the centre of ChIP-seq peaks as our TF binding sites for all downstream analyses.

We then use 10 times 10 -fold CV to evaluate the predictive ability of a RF classifiers trained on the TCF7L2 and the MAX datasets. Using the Area Under the Receiver Operating Characteristic curve (AUROC) as a measure, we found that our RF classifiers indeed has a clear ability to discriminate among the 6 cell types profiled in the TCF7L2 dataset, and among the 5 cell types profiled in the MAX dataset (Fig. 2).

To further test if the RF algorithm can distinguish different cell types based on the combinations of motifs in the $240 \mathrm{bp}$ region of the ChIP-seq peaks from the same antibody, we applied our pipeline on six additional TF datasets, each consisting of TF binding sites from five to seven cell lines (see Additional file 1). Cross-validation analysis reveals that we can use motif combinations to build a RF classifier with a reasonable sensitivity and specificity (see Additional file 4), further supporting our hypothesis that TF motif combinations are predictive of cell-type specificity.

\section{Meaningful cell-type specific motif grammars can be extracted from a RF classifier}

To identify the important features (motifs) in a trained RF classifier, we extracted the mean decrease accuracies (MDA [45]) values of all the motifs based on the RFs trained using the TCF7L2 and the MAX datasets (Fig. 3). The MDA, output by the randomForest package, informs the overall importance of a motif in the RF model. More specifically, the MDA value of a variable represents the increase in out-of-bag error that is caused by removing that variable. The MDA values reported in Fig. 3 is scaled by the standard deviation of the MDA values of all the variables. A positive MDA value indicates that inclusion of that variable is important in the RF classifier, whereas a negative MDA value indicates that inclusion of that variable negatively impact the accuracy of the RF classifier. In both TF datasets, there are a small number of motifs that have a high MDA values, suggesting that most of the discriminatory power comes from a small number of motifs (Fig. 3a and b). Using those motifs with high scaled mean decrease accuracies (with scaled MDA greater than 6), we can extract the list of motifs that are

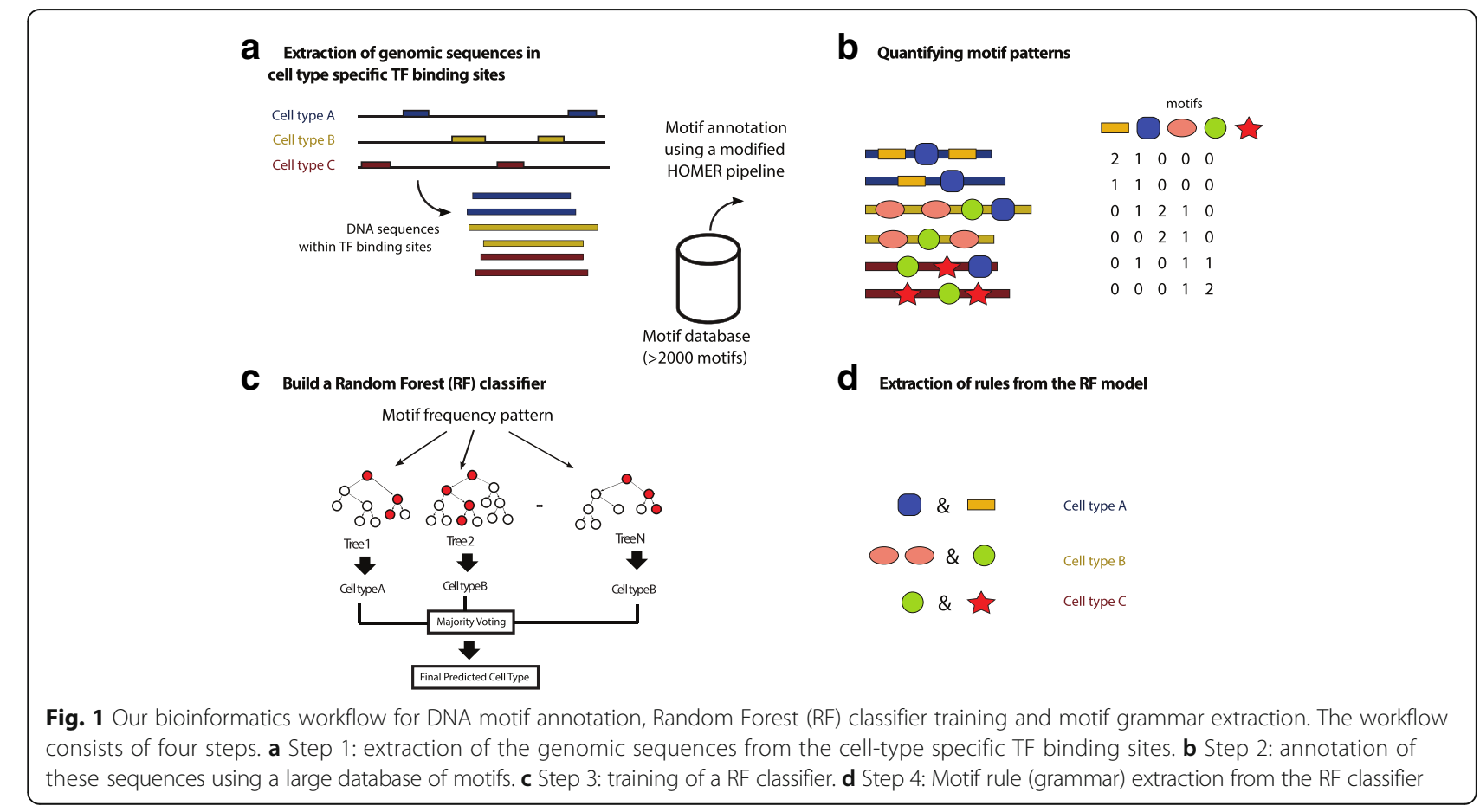


a

TCF7L2

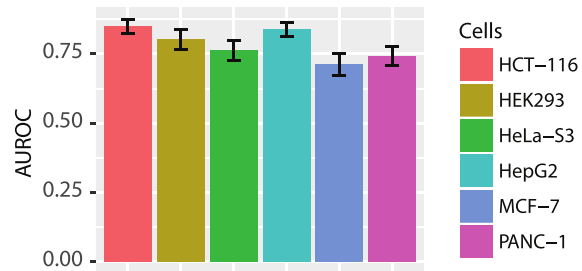

b

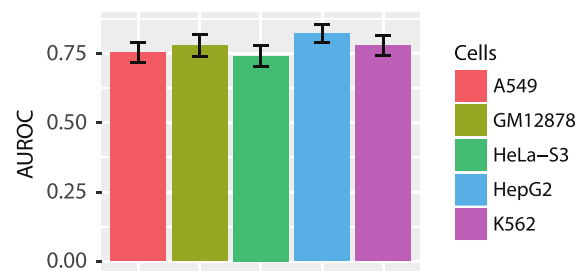

Fig. 2 The Area Under the Receiver Operator Characteristics Curve (AUROC) of the RF classifiers based on Cross-Validation. a The TCF7L2 dataset. b The MAX dataset. The error bars indicate standard deviations

cell-type specific in the two datasets (Fig. 3c and d). Only about a dozen motifs have high MDA. This finding indicates that although many motifs are present around the +/- 120 bp region of a ChIP-seq peak centre, only a small proportion of motifs show cell-type specificity. This distribution also leads to our interest to extract motif grammar rules from the RF classifiers.

\section{Discovery of motif grammar rules from random forests}

As RF has the ability to distinguish cell types based on motif occurrence in TF binding sites, we next attempt to extract interpretable rules from the trained RF classifiers. In particular, we use a rule mining approach that is implemented in an $\mathrm{R}$ package called inTrees [31], which can extract, measure, prune and select rules from a RF. The algorithm of inTrees is able to extract all the frequent decision rules from all the trees in an RF, and select set of most highly predictive and non-redundant rules based on all the training data [31]. By using the inTrees package, a number of cell-type specific rules were extracted from the two RF classifiers (Tables 1 and 2). Most of these rules are based on motifs with a high MDA value (e.g., NFE2 and

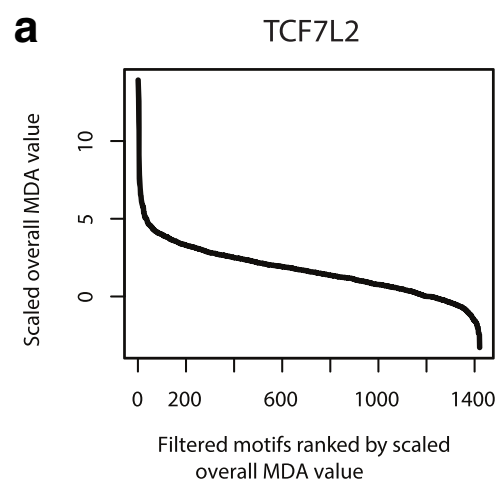

C

$$
\text { TCF7L2 }
$$

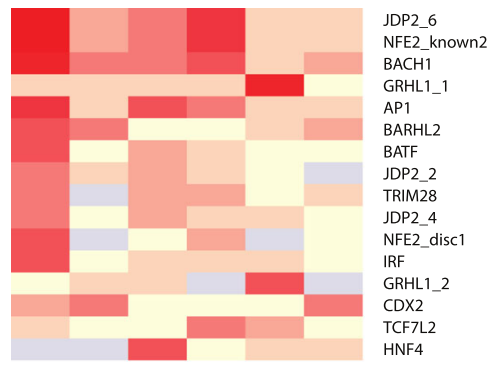

b

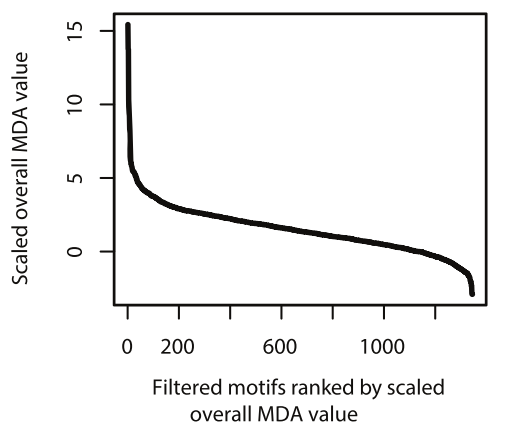

d MAX

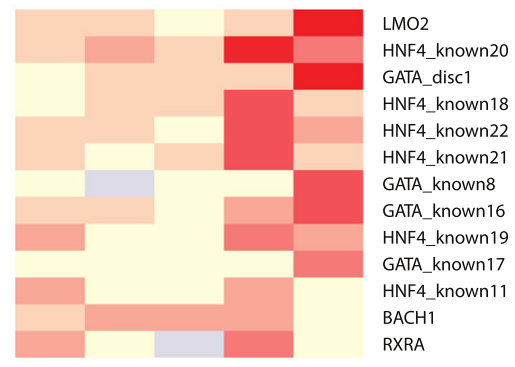

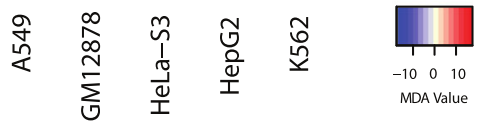

Fig. 3 The mean decrease accuracies (MDA) of motif importance extracted from the trained RF classifiers. a The sorted MDA values extracted from the TCF7L2 RF. $\mathbf{b}$ The sorted MDA values extracted from the MAX RF. $\mathbf{c}$ Heat map showing the MDA values of the top motifs in the TCF7L2 RF. $\mathbf{d}$ Heat map showing the MDA values of the top motifs in the MAX RF 
Table 1 DNA motif rules extracted from the RF classified trained on the TCF7L2 dataset

\begin{tabular}{lll}
\hline Rule & Prediction & Reference \\
\hline NFE2 $>=3$ & HCT-116 (colon cancer cells) & {$[56,57]$} \\
GSX1 $<=35 \&$ NFE2 $>=2$ & & NFE2 [56, 57] \\
BACH1 $>=2 \&$ FOXO3 $<=2 \&$ HOMEZ $<=5$ & BACH1 [55] \\
BACH1 $<=1 \&$ E2F $>=2 \&$ HOXB13 $>=3$ & HEK293 (embryonic kidney cells) & E2F [63], HOXB13 [64] \\
CDX2 $>=5 \&$ GATA $>=2 \&$ MAF $=0$ & & GATA [65, 66] \\
BACH1 $<=1 \&$ HNF4 $<=6 \&$ HOXA13 $>=3 \&$ OTX1 $>=9$ & & HOXB13 [64] \\
JDP2 $>=2 \&$ SOX21 $>=37$ & HeLa-S3 (cervical carcinoma cells) & JDP2 [54] \\
HNF4 $>=2$ & HepG2 (liver cancer cells) & [50] \\
HOXB13 $<=3 \&$ JDP2 $<=1 \&$ TCF7L2 $>=5$ & & TCF7L2 [67] \\
HNF4 $>=1 \&$ HOXC10 $<=16 \&$ SOX9 $>=5$ & & HNF4 [50], SOX9 [68] \\
GRHL1 $>=4$ & MCF-7 (mammary gland adenocarcinoma cells) & GRHL1 [58] \\
No rule identified & PANC-1 (pancreatic cancer cells) &
\end{tabular}

The numbers in the rules represent the motif frequency detected in the $+/-120$ bp regions from the peak centre

BACH1 in the TCF7L2 dataset; HNF4, AP1, LMO2 and GATA in the MAX dataset), thus supporting that inTrees are discovering important rules that are present in the RF. We found that the majority of the TFs in the extracted rule have reported cell-type or tissue-specific expression specificities, such as IRF in lymphoblastoid cell-line $[46,47]$, AP-1 in cervical cancer [48, 49], HNF4 in liver and colon cells [50], LMO2 [51] and GATA in leukaemia cells $[52,53]$. For some other motifs in the table, their TF proteins are considered as oncogenes or related to tumorigenesis (e.g., JDP2 [54], BACH1 [55] and NFE2 [56, 57]). The motif of GRHL1, whose TF is reported to interact with estrogen receptor, is also found to be expressed in mammary gland adenocarcinoma cells [58]. Our results suggest that our rule mining approach can indeed discover biologically meaningful motif rules from a RF classifier.

\section{Discussion}

Using two ENCODE TF ChIP-seq datasets, our study shows that different combination of a small number of motifs is sufficient to discriminate TF binding sites that are used in different cell types. Also, we demonstrate how we could use a Random Forest (RF) classifier for classification and rule extraction, highlighting the power of opening up a 'black box' machine learning model.

Our pipeline is unique as we are annotating TF binding site sequences with $>2000$ known motifs. The use of such as comprehensive TF motif database is important as the goal is to test the hypothesis that motif combinations alone can be predictive of cell-type-specific TF binding sites (cis regulatory elements). Nonetheless, our study is only the first step towards deciphering the DNA motif grammar. Besides motif combinations, cell-type specificity may also be affected by the spatial arrangement of the motifs, existing histone modifications and DNA accessibility $[13,18,19]$, and long range interactions [59]. Nonetheless, our finding is an important step towards discovering a cell-type specific TF motif grammar.

\section{Conclusion}

Our bioinformatics analysis supports the hypothesis that combinatorial TF motif patterns are cell-type specific.

Table 2 DNA motif rules extracted from the RF classified trained on the MAX dataset

\begin{tabular}{lll}
\hline Rule & Prediction & Reference \\
\hline IRF $>=1$ & GM12878 (lymphoblastoid cells) & {$[46,47]$} \\
JDP2 $>=1$ & HeLa-S3 (cervical carcinoma cells) & {$[54]$} \\
AP1 $>=9$ \& HESX1 $>=2 \&$ LMO2 $>=2$ & HepG2 (liver cancer cells) & AP1 [48, 49] \\
EMX1 $<=11 \&$ ETS $<=12 \&$ HNF4 $>=1$ & & HNF4 [50], ETS [69] \\
HNF4 $>=1$ \& IRF4 $<=4 \&$ RUNX2 $<=4 \&$ TAL1 $<=4$ & K562 (immortalised myelogenous leukaemia cells) & GATA [52, 53], LMO2 [51] \\
ALX3 $<=26 \&$ EVX1 $>=6 \&$ GATA $>=2 \&$ LMO2 $>=2$ & & GATA [52, 53] \\
GATA $>=4$ \& HNF4 $=0$ \& POU4F3 $<=4$ & A549 (adenocarcinomic alveolar basal epithelial cells) &
\end{tabular}




\section{Methods}

\section{Datasets}

We downloaded ChIP-seq peak files of two TFs TCF7L2 and MAX from the ENCODE ChIP-seq Experiment Matrix [60]. To maintain consistency across datasets, we only used the ChIP-seq peaks from ENCODE/SYDH standard (mapped to hg19 reference genome) by peak caller "PeakSeq1.0". For multiple entries in the ENCODE database, only IgG normalised ChIP-seq peaks were chosen.

\section{Motif annotation and random Forest implementation}

We used the $\mathrm{R}$ package randomForest [61] for the implementation of RF. We systematically evaluated how many trees are needed to train a good RF based on our two datasets. The default tree number 500 is adequate for stabilising the out of bag (OOB) error (see Additional file 5). Therefore 500 trees were used in all our analyses. The input features for the Random Forest are the top 500 strongest unique ChIP-seq peaks by $p$-value for each of the six celllines in the TCF7L2 and each of the five cell-lines in MAX datasets. Therefore, the input for each RF is a matrix of number of occurrence of 2067 motifs in 3000 (or 2500) cell-type specific TF binding sites in the TCF7L2 dataset (or the MAX dataset). Our motif database consists of 2065 motifs from ENCODE [44], as well as two de novo TCF7L2 motifs identified by Frietze et al. [11]. The motif database was then converted to the HOMER motif database format.

\section{Evaluation of classifier performance}

We have assessed the performance of the RF classifier through several methods, namely cross validation, out of bag errors and ROC curves. Cross-validations were used to estimate the classifier errors. We have performed 10fold cross-validations ten times on Amazon AWS using the R packages foreach, doMC and caret. Out of bag errors make use of the unselected samples in each tree in the forest to estimate the classifier errors, and have been shown accurate empirically [62]. Besides estimating classifier errors, we have also calculated AUROC values to assess the performance of the RF classifiers. More specifically, a binary classifier for each cell-line can be obtained as follows: for each sample, the RF classifier outputs the percentage of decision trees that predict each cell-line, and this percentage is used as the discrimination threshold of a binary classifier for the cellline. The AUROC values for each of these binary classifiers are shown in Fig. 2.

\section{Identification of cell-type discriminatory motifs in a RF classifier}

Two independent methods were used to identify celltype discriminatory motifs. Firstly, the mean decrease accuracy (MDA), output by the randomForest package, informs the overall importance of a motif in the RF model. Specifically, the RF training was performed in two rounds. In the first round, features with negative MDAs were removed so that these irrelevant features were not present in the final random forest. Then, we optimised the number of features in each decision tree using the 'tuneRF' function in the randomForest package by minimizing the out-of-bag errors. More specifically, through out-of-bag error estimation, it estimates the loss of accuracy after randomly permuting the values of each motif. We then plot the MDA of all the motifs in Fig. 3.

A second method is to extract frequent and important rules present in the decision trees in an RF, using the $R$ package inTrees. For this procedure, we used RFs trained from the two whole datasets as the inputs. Rules were extracted and pruned using the 'getRuleMetric' and 'pruneRule' functions respectively. Then a set of relevant and non-redundant rules were selected using regularised RFs through the 'selectRuleRRF' function, after which the rules were further selected based on the frequency and error - rules with a frequency below $8 \%$ or error above 0.7 were eliminated. The resulting selected rules are included in Tables 1 and 2.

\section{Additional files}

Additional file 1: Table S1. Datasets used in this study. The ENCODE data we used in this study with the information of TFs, cell-lines, reference genome, peak caller information and the GEO Accession ID. (XLS $42 \mathrm{~kb}$ )

Additional file 2: Figure S1. $-\log 10 p$-value distribution of the peaks on the TCF7L2 and the MAX datasets. The positions of the 500th peak in each plot ranked by p-value were highlighted in red lines. (PDF 343 kb)

Additional file 3: Figure S2. - Cross validation of RF classifiers trained on the TCF7L2 and the MAX datasets. We employed F1 score $(F 1=2$ * precision * recall / (precision + recall)) to measure the performance of the RF classifier for each cell line. We evaluated the discriminatory power of TF binding sites that are +/- $5 \mathrm{bp}$ to $+/-300 \mathrm{bp}$ from the centre of each of the TF ChIP-seq peak. (PDF $29 \mathrm{~kb}$ )

Additional file 4: Figure S3. - The Area Under the Receiver Operator Characteristics Curve (AUROC) of the RF classifiers based on cross-validation. (A) The CEBPB dataset. (B) The CHD2 dataset. (C) The EP300 dataset. (D) The JUND dataset. (E) The MXI1 dataset. (F) The RAD21 dataset. Error bars indicate standard deviations. (PDF $157 \mathrm{~kb}$ )

Additional file 5: Figure S4. - Out of Bag (OOB) curves of the RF of TCF7L2 dataset and MAX dataset. From the curve of the OOB error rate against the number of trees, we find that 500 trees are sufficient to minimise errors, and increasing the number of trees would not help the RF classifier to perform better. We used 500 trees in our downstream analyses. (PDF $18 \mathrm{~kb}$ )

\section{Abbreviations}

AUROC: Area under Receiver Operating Characteristic curve; ChIP-seq: Chromatin Immuno-Precipitation Sequencing; CV: Cross-Validation; ENCODE: Encyclopedia of DNA Elements; HOMER: Hypergeometric Optimization of Motif EnRichment; MAX: Myc-associated factor X; MDA: Mean Decrease Accuracy; RF: Random Forest; ROC curve: Receiver Operating Characteristic curve; TCF7L2: Transcription factor 7-like 2; TF: Transcription Factor

Acknowledgements

We thank Joseph Godbehere and Ralph Patrick for their technical assistance. 


\section{Funding}

This work is supported in part by the New South Wales Ministry of Health, the Human Frontier Science Program (RGY0084/2014), the National Health and Medical Research Council of Australia (1105271), the National Heart Foundation of Australia (100848), and the Amazon Web Services (AWS) Cloud Credits for Research. Publication of this article was funded by the Victor Chang Cardiac Research Institute.

\section{Availability of data and materials}

Project name: motif-discovery-pipeline.

Project home page: https://github.com/NCCRI/motif-discovery-pipeline Archived version: https://github.com/NCCRI/motif-discovery-pipeline/releases/ $\operatorname{tag} / 1.01$

Operating system(s): Linux system

Programming language: Perl, bash and $\mathrm{R}$.

Other requirements: HOMER 4.9 or higher need to be successfully installed first, with the Homo sapiens reference genome hg19 also downloaded by HOMER configuration script.

License: FreeBSD.

\section{About this supplement}

This article has been published as part of BMC Genomics Volume 19 Supplement 1, 2018: 16th International Conference on Bioinformatics (InCoB 2017): Genomics. The full contents of the supplement are available online at https://bmcgenomics.biomedcentral.com/articles/supplements/ volume-19-supplement-1.

\section{Authors' contributions}

XW and JWKH conceived the idea. XW designed and implemented the software pipeline. XW and PL conducted the study. All authors wrote, read, and approved the final version of the manuscript.

\section{Ethics approval and consent to participate}

Not applicable.

\section{Consent for publication}

Not applicable.

\section{Competing interests}

The authors declare that they have no competing interests.

\section{Publisher's Note}

Springer Nature remains neutral with regard to jurisdictional claims in published maps and institutional affiliations.

\section{Published: 19 January 2018}

\section{References}

1. Spitz F, Furlong EEM. Transcription factors: from enhancer binding to developmental control. Nat Rev Genet. 2012;13:613-26.

2. Chen $X, X u H$, Yuan $P$, Fang F, Huss M, Vega VB, et al. Integration of external signaling pathways with the core transcriptional network in embryonic stem cells. Cell. 2008;133:1106-17.

3. Kaur G, Costa MW, Nefzger CM, Silva J, Fierro-González JC, Polo JM, et al. Probing transcription factor diffusion dynamics in the living mammalian embryo with photoactivatable fluorescence correlation spectroscopy. Nat Commun. 2013:4:1637.

4. Tsankov AM, Gu H, Akopian V, Ziller MJ, Donaghey J, Amit I, et al. Transcription factor binding dynamics during human ES cell differentiation. Nature. 2015;518:344-9.

5. Bertoli C, Skotheim JM, de Bruin RAM. Control of cell cycle transcription during G1 and S phases. Nat Rev Mol Cell Biol. 2013;14:518-28.

6. MacAlister CA, Ohashi-Ito K, Bergmann DC. Transcription factor control of asymmetric cell divisions that establish the stomatal lineage. Nature. 2007:445:537-40.

7. MacArthur S, Li X-Y, Li J, Brown JB, Chu HC, Zeng L, et al. Developmental roles of 21 drosophila transcription factors are determined by quantitative differences in binding to an overlapping set of thousands of genomic regions. Genome Biol. 2009;10:R80.

8. Medzhitov R, Horng T. Transcriptional control of the inflammatory response. Nat Rev Immunol. 2009;9:692-703.
9. Ueda HR, Chen W, Adachi A, Wakamatsu H, Hayashi S, Takasugi T, et al. A transcription factor response element for gene expression during circadian night. Nature. 2002;418:534-9.

10. Hanna JH, Saha K, Jaenisch R. Pluripotency and cellular reprogramming: facts, hypotheses, unresolved issues. Cell. 2010;143:508-25.

11. Frietze $S$, Wang R, Yao L, Tak YG, Ye Z, Gaddis M, et al. Cell type-specific binding patterns reveal that TCF7L2 can be tethered to the genome by association with GATA3. Genome Biol. 2012;13:R52.

12. Mullen AC, Orlando DA, Newman JJ, Lovén J, Kumar RM, Bilodeau S, et al. Master transcription factors determine cell-type-specific responses to TGF- $\beta$ signaling. Cell. 2011;147:565-76.

13. Yáñez-Cuna JO, Kvon EZ, Stark A. Deciphering the transcriptional cis-regulatory code. Trends Genet. 2013:29:11-22.

14. Cai Y, Hartnett B, Gustafsson C, Peccoud JA. Syntactic model to design and verify synthetic genetic constructs derived from standard biological parts. Bioinformatics. 2007;23:2760-7.

15. Purcell O, Peccoud J, Lu TK. Rule-Based Design of Synthetic Transcription Factors in eukaryotes. ACS Synth Biol. 2014;3:737-44.

16. Guo Y, Mahony S, Gifford DK. High resolution genome wide binding event finding and motif discovery reveals transcription factor spatial binding constraints. PLoS Comput Biol. 2012;8:e1002638.

17. Heinz S, Benner C, Spann N, Bertolino E, Lin YC, Laslo P, et al. Simple combinations of lineage-determining transcription factors prime cis-regulatory elements required for macrophage and B cell identities. Mol Cell. 2010;38:576-89.

18. Wang J, Zhuang J, lyer S, Lin X, Whitfield TW, Greven MC, et al. Sequence features and chromatin structure around the genomic regions bound by 119 human transcription factors. Genome Res. 2012;22:1798-812.

19. Arvey A, Agius P, Noble WS, Leslie C. Sequence and chromatin determinants of cell-type-specific transcription factor binding. Genome Res. 2012;22:1723-34.

20. Ha M, Hong SDNA. Context represents transcription regulation of the gene in mouse embryonic stem cells. Sci Rep. 2016;6:24343.

21. Alipanahi B, Delong A, Weirauch MT, Frey BJ. Predicting the sequence specificities of DNA- and RNA-binding proteins by deep learning. Nat Biotechnol. 2015:33:831-8.

22. Ho, T.K. Random Decision Forest. In Proceedings of the Third International Conference on Document Analysis and Recognistion. 1995;278-82.

23. Ho TK. The random subspace method for constructing decision forests. IEEE Trans Pattern Anal Mach Intell. 1998;20:832-44.

24. Hastie T, Tibshirani R and Friedman J. The Elements of Statistical Learning. New York: Springer; 2009.

25. Díaz-Uriarte R, Alvarez de Andrés S. Gene selection and classification of microarray data using random forest. BMC Bioinformatics. 2006;7:3.

26. Christensen BC, Houseman EA, Godleski JJ, Marsit CJ, Longacker JL, Roelofs $\mathrm{CR}$, et al. Epigenetic profiles distinguish pleural mesothelioma from normal pleura and predict lung asbestos burden and clinical outcome. Cancer Res. 2009;69:227-34.

27. De Lobel L, Geurts P, Baele G, Castro-Giner F, Kogevinas M, Van Steen KA. Screening methodology based on random forests to improve the detection of gene-gene interactions. Eur J Hum Genet. 2010;18:1127-32.

28. Caporaso JG, Lauber CL, Costello EK, Berg-Lyons D, Gonzalez A, Stombaugh J, et al. Moving pictures of the human microbiome. Genome Biol. 2011;12:R50.

29. Rödelsperger C, Guo G, Kolanczyk M, Pletschacher A, Köhler S, Bauer S, et al. Integrative analysis of genomic, functional and protein interaction data predicts long-range enhancer-target gene interactions. Nucleic Acids Res. 2011;39:2492-502.

30. Mashayekhi M., Gras R. Rule Extraction from Random Forest: the RF+HC Methods. In Advances in Artificial Intelligence. 2015; Lecture Notes in Computer Science vol 9091, 223-237, Springer, Cham, Switzerland.

31. Deng H. Interpreting tree ensembles with inTrees. ArXiv. 2014;1408:5456.

32. The ENCODE Project Consortium. An integrated encyclopedia of DNA elements in the human genome. Nature. 2012;489:57-74

33. Ravindranath $\mathrm{A}$, O'Connell A, Johnston PG, El-Tanani MK. The role of LEF/TCF factors in neoplastic transformation. Curr Mol Med. 2008;8:38-50.

34. Grove EA. Wnt signaling meets internal dissent. Genes Dev. 2011:25:1759-62.

35. Jin T, Liu L. The Wnt signaling pathway effector TCF7L2 and type 2 diabetes mellitus. Mol Endocrinol Baltim Md. 2008:22:2383-92.

36. Weedon MN. The importance of TCF7L2. Diabet Med J Br Diabet Assoc. 2007:24:1062-6.

37. Norton L, Fourcaudot M, Abdul-Ghani MA, Winnier D, Mehta FF, Jenkinson CP, et al. Chromatin occupancy of transcription factor 7-like 2 (TCF7L2) and its role in hepatic glucose metabolism. Diabetologia. 2011;54:3132-42. 
38. Zhao J, Schug J, Li M, Kaestner KH, Grant SFA. Disease-associated loci are significantly over-represented among genes bound by transcription factor 7-like 2 (TCF7L2) in vivo. Diabetologia. 2010;53:2340-6.

39. Trompouki E, Bowman TV, Lawton LN, Fan ZP, D-C W, DiBiase A, et al. Lineage regulators direct BMP and Wnt pathways to cell-specific programs during differentiation and regeneration. Cell. 2011;147:577-89.

40. Amati B, Land H. Myc-max-mad: a transcription factor network controlling cell cycle progression, differentiation and death. Curr Opin Genet Dev. 1994;4:102-8.

41. FitzGerald MJ, Arsura M, Bellas RE, Yang W, Wu M, Chin L, et al. Differential effects of the widely expressed dMax splice variant of max on E-box vs initiator element-mediated regulation by c-Myc. Oncogene. 1999;18:2489-98.

42. Romero OA, Torres-Diz M, Pros E, Savola S, Gomez A, Moran S, et al. MAX inactivation in small cell lung cancer disrupts MYC-SWI/SNF programs and is synthetic lethal with BRG1. Cancer Discov. 2014:4:292-303.

43. Rudin CM, Poirier JTMYC. MAX, and small cell lung cancer. Cancer Discov. 2014:4:273-4.

44. Kheradpour P, Kellis M. Systematic discovery and characterization of regulatory motifs in ENCODE TF binding experiments. Nucleic Acids Res. 2014;42:2976-87.

45. Nicodemus KK. Letter to the editor: on the stability and ranking of predictors from random forest variable importance measures. Brief Bioinform. 2011;12:369-73.

46. Xu D, Zhao L, Del Valle L, Miklossy J, Zhang L. Interferon regulatory factor 4 is involved in Epstein-Barr virus-mediated transformation of human B lymphocytes. J Virol. 2008;82:6251-8.

47. Tzellos S, Correia PB, Karstegl CE, Cancian L, Cano-Flanagan J, McClellan MJ, et al. A single amino acid in EBNA-2 determines superior B Lymphoblastoid cell line growth maintenance by Epstein-Barr virus type 1 EBNA-2. J Virol. 2014:88:8743-53

48. Prusty BK, Das BC. Constitutive activation of transcription factor AP-1 in cervical cancer and suppression of human papillomavirus (HPV) transcription and AP-1 activity in HeLa cells by curcumin. Int J Cancer. 2005;1 13:951-60.

49. Mahata S, Bharti AC, Shukla S, Tyagi A, Husain SA, Das BC. Berberine modulates AP-1 activity to suppress HPV transcription and downstream signaling to induce growth arrest and apoptosis in cervical cancer cells. Mol Cancer. 2011;10:39.

50. Babeu J-P, Boudreau F. Hepatocyte nuclear factor 4-alpha involvement in liver and intestinal inflammatory networks. World J Gastroenterol WJG. 2014;20:22-30.

51. McCormack MP, Young LF, Vasudevan S, de Graaf CA, Codrington R, Rabbitts TH, et al. The Lmo2 oncogene initiates leukemia in mice by inducing thymocyte self-renewal. Science. 2010;327:879-83.

52. Yang LV, Wan J, Ge Y, Fu Z, Kim SY, Fujiwara Y, et al. The GATA site-dependent hemogen promoter is transcriptionally regulated by GATA1 in hematopoietic and leukemia cells. Leukemia. 2006;20:417-25

53. Ayala RM, Martínez-López J, Albízua E, Diez A, Gilsanz F. Clinical significance of Gata-1, Gata-2, EKLF, and c-MPL expression in acute myeloid leukemia. Am J Hematol. 2009:84:79-86.

54. Tanigawa S, Lee CH, Lin CS, Ku CC, Hasegawa H, Qin S, et al. Jun dimerization protein 2 is a critical component of the Nrf2/MafK complex regulating the response to ROS homeostasis. Cell Death Dis. 2013;4:e921.

55. Cantor SB, Bell DW, Ganesan S, Kass EM, Drapkin R, Grossman S, et al. BACH1, a novel helicase-like protein, interacts directly with BRCA1 and contributes to its DNA repair function. Cell. 2001;105:149-60.

56. Kashif M, Hellwig A, Hashemolhosseini S, Kumar V, Bock F, Wang H, et al. Nuclear factor erythroid-derived 2 (Nfe2) regulates JunD DNA-binding activity via acetylation: a novel mechanism regulating trophoblast differentiation. J Biol Chem. 2012;287:5400-11.

57. Gasiorek JJ, Blank V. Regulation and function of the NFE2 transcription factor in hematopoietic and non-hematopoietic cells. Cell Mol Life Sci CMLS. 2015;72:2323-35.

58. Zheng $Y$, Shao $X$, Huang $Y$, Shi L, Chen B, Wang $X$, et al. Role of estrogen receptor in breast cancer cell gene expression. Mol Med Rep. 2016;13:4046-50.

59. Guo Y, Gifford DK. Modular combinatorial binding among human trans-acting factors reveals direct and indirect factor binding. BMC Genomics. 2017;18:45.

60. ENCODE ChIP-seq Experiment Matrix [Internet]. [cited Sep 6, 2017]. Available from: http://genome.ucsc.edu/encode/dataMatrix/ encodeChipMatrixHuman.html

61. Liaw A, Wiener M. Classification and regression by randomForest. R News. 2002;23:18-22
62. Breiman L. Out-of-bag estimation. Technical report. U.C. Berkeley: Department of Statistics; 1996.

63. Li J, Ran C, Li E, Gordon F, Comstock G, Siddiqui H, et al. Synergistic function of E2F7 and E2F8 is essential for cell survival and embryonic development. Dev Cell. 2008;14:62-75.

64. Okuda H, Toyota M, Ishida W, Furihata M, Tsuchiya M, Kamada M, et al. Epigenetic inactivation of the candidate tumor suppressor gene HOXB13 in human renal cell carcinoma. Oncogene. 2006;25:1733-42

65. Oosterwegel M, Timmerman J, Leiden J, Clevers $\mathrm{H}$. Expression of GATA-3 during lymphocyte differentiation and mouse embryogenesis. Dev Immunol. 1992;3:1-11.

66. Debacker C, Catala M, Labastie M-C. Embryonic expression of the human GATA-3 gene. Mech Dev. 1999;85:183-7.

67. Neve B, Bacquer OL, Caron S, Huyvaert M, Leloire A, Poulain-Godefroy O, et al. Alternative human liver transcripts of TCF7L2 bind to the gluconeogenesis regulator HNF4a at the protein level. Diabetologia. 2014;57:785-96.

68. Guo X, Xiong L, Sun T, Peng R, Zou L, Zhu H, et al. Expression features of SOX9 associate with tumor progression and poor prognosis of hepatocellular carcinoma. Diagn Pathol. 2012;7:44.

69. Knittel T, Kobold D, Dudas J, Saile B, Ramadori G. Role of the Ets-1 transcription factor during activation of rat hepatic stellate cells in culture. Am J Pathol. 1999:155:1841-8.

\section{Submit your next manuscript to BioMed Central and we will help you at every step:}

- We accept pre-submission inquiries

- Our selector tool helps you to find the most relevant journal

- We provide round the clock customer support

- Convenient online submission

- Thorough peer review

- Inclusion in PubMed and all major indexing services

- Maximum visibility for your research

Submit your manuscript at www.biomedcentral.com/submit
) Biomed Central 